\title{
Insight into the Binding Mode of Agonists of the Nicotinic Acetylcholine Receptor from Calculated Electron Densities
}

\author{
Michael E. Beck, ${ }^{*[a]}$ Oliver Gutbrod, ${ }^{[b]}$ and Svend Matthiesen ${ }^{[b]}$
}

Insect nicotinic acetylcholine receptors ( $n$ AChRs) are among
the most prominent and most economically important insecti-
cide targets. Thus, an understanding of the modes of binding
of respective agonists is important for the design of specific
compounds with favorable vertebrate profiles. In the case of
$n A C h R s$, the lack of available high-resolution X-ray structures
leaves theoretical considerations as the only viable option.
Starting from classical homology and docking approaches,
binding mode hypotheses are created for five agonists of the

$n A C h R$, covering insecticides in the main group 4 of the Insecticide Resistance Action Committee (IRAC) mode of action (MoA) classification, namely, neonicotinoids, nicotine, sulfoxaflor, and butenolides. To better understand these binding modes, the topologies of calculated electron densities of small-model systems are analyzed in the framework of the quantum theory of atoms in molecules. The theoretically obtained modes of binding are very much in line with the biology-driven IRAC MoA classification of the investigated ligands.

\section{Introduction}

\subsection{The Nicotinic Acetylcholine Receptor and Its Agonists}

The nicotinic acetylcholine receptor $(n A C h R)$ is a cation-selective ligand-gated ion channel belonging to the "Cys-loop" superfamily. It is a large, pentameric transmembrane protein complex (approximately $290 \mathrm{kDa}$ ). It plays a central role in rapid signal transduction by gating ions elicited by acetylcholine at the vertebrate neuromuscular junction as well as in all animal central and peripheral nervous systems..$^{[1-3]}$

$n A C h R s$ are homo- and heteromeric pentamers consisting of structurally closely related subunits, each comprising approximately 500 amino acid residues. The extracellular, N-terminal domains are involved in ligand binding and are made up of six distinct regions (called loops A-F), as well as a Cys-Cys loop. The actual cation channel is formed by four transmembranespanning domains at the $\mathrm{C}$ terminus called TM1 to TM4. An intracellular region extends from TM3 and TM4. ${ }^{[4]}$ If viewed along the axis of the central pore, the pentameric arrangement is approximately $C_{5}$ symmetric.

[a] Dr. M. E. Beck

Head of Computational Science

Bayer CropScience AG, R\&D-SMR-RT-Computational Science, Alfred-Nobel-Strasse 50, 40789 Monheim am Rhein (Germany) E-mail:michael.beck@bayer.com

[b] Dr. O. Gutbrod, S. Matthiesen

Molecular Modelling

Bayer CropScience AG, R\&D-SMR-RT-Computational Science, Alfred-Nobel-Strasse 50, 40789 Monheim am Rhein (Germany)

Supporting Information for this article is available on the WWW under http://dx.doi.org/10.1002/cphc.201500341.

of (c) 2015 The Authors. Published by Wiley-VCH Verlag GmbH \& Co. KGaA. This is an open access article under the terms of the Creative Commons Attribution Non-Commercial NoDerivs License, which permits use and distribution in any medium, provided the original work is properly cited, the use is non-commercial and no modifications or adaptations are made.

In vertebrates, a diverse family of $n A C h R$ subtypes is generated from pentameric co-assemblies from 17 known nAChR subunits $\left(\alpha_{1}-\alpha_{10}, \beta_{1}-\beta_{4}, \gamma, \delta\right.$, and $\left.\varepsilon\right)$. In insects, knowledge is more limited, but a multitude of genes has been identified encoding several $n A C h R$ subunits. As in vertebrates, the existence of a wide range of insect $n A C h R$ subtypes across species is highly likely. ${ }^{[5]}$ For example, 10 subunits are known in the fly Drosophila melanogaster ${ }^{[6]}\left(D \alpha_{1-7}\right.$ and $\left.\mathrm{D} \beta_{1-3}\right)$, also 10 in the mosquito Anopheles gambiae (Agam $\alpha_{1-9}$ and Agam $\left.\beta_{1}\right)$, but 11 in the honey bee Apis mellifera (Amel $\alpha_{1-9}$ and Amel $\left.\beta_{1,2}\right) .^{[7]}$ However, architecture, diversity, tertiary structure, as well as molecular function of native insect $n A C h R s$ are poorly understood compared to the vertebrate receptors. ${ }^{[8]}$ The clear implications for homology modeling are discussed later.

This lack of knowledge stands in sharp contrast to the economic importance of insect $n A C h R s$ as major targets for insecticide action. nAChRs are of key importance in insect central nervous systems (CNSs). In 2011, world-wide sales of agonists of $n A C h R$ as insecticides exceeded 3.5 billion USD, accounting for $29 \%$ of the total insecticide world market. ${ }^{[9]}$ Despite some public debate on neonicotinoid bee safety, particularly in Europe ${ }^{[10]}$ agonists of insect $n A C h R s$, including the chemical subclass of neonicotinoids, remain not only effective insecticides but show pronouncedly favorable safety profiles. ${ }^{[11-13]}$ Historically, nicotine was the first commercially used insecticide as an active $n A C h R$ agonist. However, due to its high mammalian acute toxicity by all routes of exposure (oral, dermal, and inhalation ${ }^{[14]}$ and the introduction of toxicologically more benign insecticides, its use declined steadily to almost zero decades ago. The Insecticide Resistance Action Committee (IRAC, http://www.irac-online.org/) working under the auspices of CropLife International is considered the global authority in insecticide mode of action (MoA) classification for resistance management purposes. ${ }^{[15]}$ In this classification, main group 4 
Table 1. IRAC MoA classification: agonists of the $n A C h R$ (main group 4).

\begin{tabular}{|c|c|c|}
\hline \multicolumn{2}{|c|}{ Chemical subgroups } & \multirow{2}{*}{$\begin{array}{l}\text { Representative compounds } \\
\text { imidacloprid (4), thiacloprid (2) }\end{array}$} \\
\hline $4 \mathrm{~A}$ & neonicotinoids & \\
\hline $4 B$ & nicotine & protonated nicotine (5) \\
\hline $4 C$ & sulfoxaflor & sulfoxaflor (1) \\
\hline $4 \mathrm{D}$ & butenolides & flupyradifurone (3) \\
\hline
\end{tabular}<smiles>CC(c1ccc(C(F)(F)F)nc1)S(C)(=O)=NC#N</smiles>

(1)<smiles>O=C1C=C(N(Cc2ccc(Cl)nc2)CC(F)F)CO1</smiles><smiles>N#CN=C1SCCN1Cc1ccc(Cl)nc1</smiles>

(2)<smiles>O=[N+]([O-])/N=C1\NCCN1Cc1ccc(Cl)nc1</smiles>

(4)

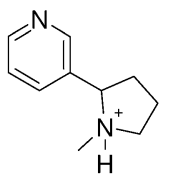

(5)

Figure 1. Agonists of the $n A C h R$ investigated in this study.

combines $n A C h R$ agonists, chemically subclassified as outlined in Table 1. In this contribution, the chemical subgroup $4 \mathrm{~A}$, the neonicotinoids, is represented by two selected members, imidacloprid (2) and thiacloprid (4, see Figure 1). Subgroup 4B, the nicotine class, is represented by nicotine itself in its protonated form (5), which is assumed to be the actual active ingredient. Recent innovation in the field of $n A C h R$ agonists is reflected by the introduction of subgroup $4 \mathrm{C}$, sulfoxaflor $(1),{ }^{[16]}$ and subgroup 4D, butenolides, represented by flupyradifurone (3). ${ }^{[11,17,18]}$

Although the IRAC MoA classification is driven by target site and biological considerations to provide guidance in effective and sustainable insecticide resistance management strategies rather than by chemical and biophysical thinking, we will show in this study, that the IRAC subclassification in group 4 is also reflected in physical terms, namely, the Laplacian of the electron density.

\subsection{Binding Mode Hypothesis Generation}

$n A C h R s$ share the fate of many other integral membrane proteins, in that it has not yet been possible to conduct high-resolution X-ray crystallographic studies; single particle electron microscopy studies are also not available for this receptor. An important breakthrough in the understanding of $n A C h R s$ and agonist binding modes in particular came with the discovery, characterization, and structural determination of various acetylcholine binding proteins (AChBPs). Not only are these proteins soluble and homopentameric, they also share the same overall architecture as the extracellular portion of the $n \mathrm{AChR}^{[19]}$ which happens to host the binding site for agonists. AChBPs are found in the central nervous system of, for example, the freshwater snail Lymnea stagnalis (Ls-AChBP) ${ }^{[20]}$ and the seawater mollusc Aplysia californica (Ac-AChBP). These two AChBPs share $33 \%$ identity in their amino acid sequence. ${ }^{[21,22]}$ Moreover, not only apo structures, but a wealth of high-resolution structures of AChBPs in complex with numerous ligands, among them nicotine (5), imidacloprid (2), and thiacloprid (4), are available (see below). The striking functional and structural similarity of the Acetylcholin Binding Proteins to the nAChRs has early been recognized as particularly useful to construct homology models for extracellular domains of nAChRs by providing both an understanding of ligand-receptor interaction as well as a basis for the further rational design of new agonists. ${ }^{[11,21,23,24]}$

The difference between sensitive and nonsensitive mutants of $n A C h R$, as well as a comparison of the binding modes of 4 and 5 in $n \mathrm{AChR}$ and AChBP from Lymnea stagnalis and Aplysia californica, can be found in Ref. [12] itself and in the references cited therein. Herein, we focus on sensitive insect $n A C h R s$.

\subsection{Beyond Docking Poses}

Docking and rational design are now well-established tools for the understanding of binding modes, the generation of respective binding mode hypotheses, and, subsequently, rational design. ${ }^{[25]}$ Also in this study, this is the starting point for the discussion.

Docking poses are often remarkably accurate, although accurate prediction of protein-ligand interaction energies remains a challenge. ${ }^{[2]}$ Energies are anyway of limited use for the design and optimization of new compounds, as chemists and chemical biologists think in (3D) topologies of compounds rather than in energies.

Interactions between the participating atoms are in many cases classified only in terms of atomic distances and angular relationships. Although this is a very valid, pragmatic approach, a more robust physical appraisal would quite probably be beneficial.

It is thus tempting to dig deeper into binding mode poses as derived from docking studies and X-ray crystallography by analyzing the topology of binding of a protein-ligand complex. In this study, we use Bader's approach of the quantum theory of atoms in molecules (QTAIM) for that purpose. To arrive at an approach also feasible in an industrial setting, all QTAIM calculations were done with small-model systems rather than full active sites. Although the latter is, as of today, also possible, ${ }^{[27]}$ analysis of such calculations is cumbersome and does not provide information that can easily be translated into chemistry. QTAIM of small-model systems, however, leads to results that are understood by agrochemists and may serve as sources to inspire synthesis ideas.

\subsection{Recap of QTAIM}

It is amazing to see that Lewis formulae do not only constitute a convenient formalism, but contain more physical truth than 
their inventor possibly imagined. ${ }^{[28]}$ Especially through the work of Yang and Parr, ${ }^{[29]}$ density functional theory (DFT) and conceptual DFT have provided a solid theoretical foundation for a number of chemical concepts, such as, for example, chemical reactivity and frontier orbital theory. ${ }^{[30,31]}$ It was Bader who realized that the topology of the electron density, more precisely the Laplacian of the electron density, resembles the Lewis structure and provides further insight based on basic mathematics. ${ }^{[32,33]}$ Applications of QTAIM for biological systems can be found in Ref. [34] Here, only a very rough sketch of QTAIM is provided.

The topology of the electron density $\rho$ may be characterized in terms of its critical points (CPs), which satisfy $\nabla \rho=0$. As $\rho=$ $\rho(x, y, z)$ is a 3D function of space, there are four different kinds of critical points: nuclear critical points (NCPs), for which all three curvatures are negative; bond critical points (BCPs), with two negative curvatures and one positive curvature; ring critical points (RCPs), with one negative curvature and two positive curvatures; and finally, cage critical points (CCPs), with three positive curvatures. NCPs occur exactly at the positions of the nuclei. BCPs are saddle points in the classical sense. As the name suggests, BCPs are associated with chemical bonds. RCPs typically reside near the center of rings of atoms, whereas CCPs are found near the center of cages of atoms. Gradient paths through $\rho(x, y, z)$ (so-called trajectories) connect the critical points. A graphical representation of the gradient paths involving only NCPs and BCPs is confusingly similar to an ordinary modeling picture of the same molecule; just compare Figures 6 and 7.

A number of helpful properties can be calculated at CPs; only the charge density will be used here. It has been shown that the sum over charge densities of CPs can be correlated to bond strengths, also in weakly bound systems, ${ }^{[34]}$ including hydrogen-bonded systems. ${ }^{[35,36]}$ In the context of this study, we would also like to mention that BCP and CCP data in particular have been shown to provide good description of $\pi-\pi$ stacking interactions. ${ }^{[37]}$

\section{Results and Discussion}

\subsection{Creation of a Model of Sensitive Insect nAChR}

The models used herein were constructed from the crystal structure of Ls-AChBP (PDB-ID 119B) following a procedure similar to that reported in Ref. [40] and building on our own previous work. ${ }^{[11]}$ The exact composition of subunits of the sensitive insect $n A C h R s$ remains unknown; thus, we decided to deduce a consensus model comprising combinations of all five $\alpha$ subunits known in green peach aphid (Myzus persicae Sulzer) $n A C h R$ with one $\beta 1$ subunit each. We arrived at the final model by structure-based alignment onto a dimer extracted from the homopentameric AChBP from Lymnea Stagnalis in complex with imidacloprid (4) and another neonicotinoid insecticide, clothianidin, as well as AChBP from Aplysia californica in complex again with imidacloprid (4) and thiacloprid (2) (PDB-IDs 2ZJU, 2ZJV, 3C79, and 3C84). ${ }^{[33,54]}$

\subsection{Binding Mode Hypothesis}

Agonists $\mathbf{1}$ to $\mathbf{5}$ were manually docked into the binding site in the dimer model of the sensitive wildtype aphid receptor by using respective poses in the Lymnea stagnalis and Aplysia californica AChBP X-ray co-crystal structures as templates. As for agonists $\mathbf{1}$ and $\mathbf{3}$, no immediate template was available; as such, these two were subjected to conformational searching by using a mixed approach of simulated annealing and Monte Carlo $^{[42]}$ prior to matching them to the poses of $\mathbf{2}$ and $\mathbf{4}$, as obtained from co-crystal structures mentioned above.

Figure 2 illustrates the binding mode hypotheses thus obtained. There are three main anchoring interactions. First, the pyridine nitrogen atom present in all five agonists forms a water-mediated $\mathrm{H}$-bond to the backbone of the $\mathrm{D}$ loop of the $\beta$ subunit. Second, a cluster of conserved aromatic residues surrounds and stabilizes the $\mathrm{N}$-cyansulfoximine moiety in sulfoxaflor (1), the 2-(N-cyanimino)thiazolidine system of thiacloprid (2), the 4-aminofuran-2(5H)-one system of flupyradifurone (3), the 2-( $N$-nitroimino)imidazolidine moiety of imidacloprid (4), and the protonated $\mathrm{N}$-methylpyrrolidine moiety of nicotine (5). Finally, there is a key interaction of a charged arginine
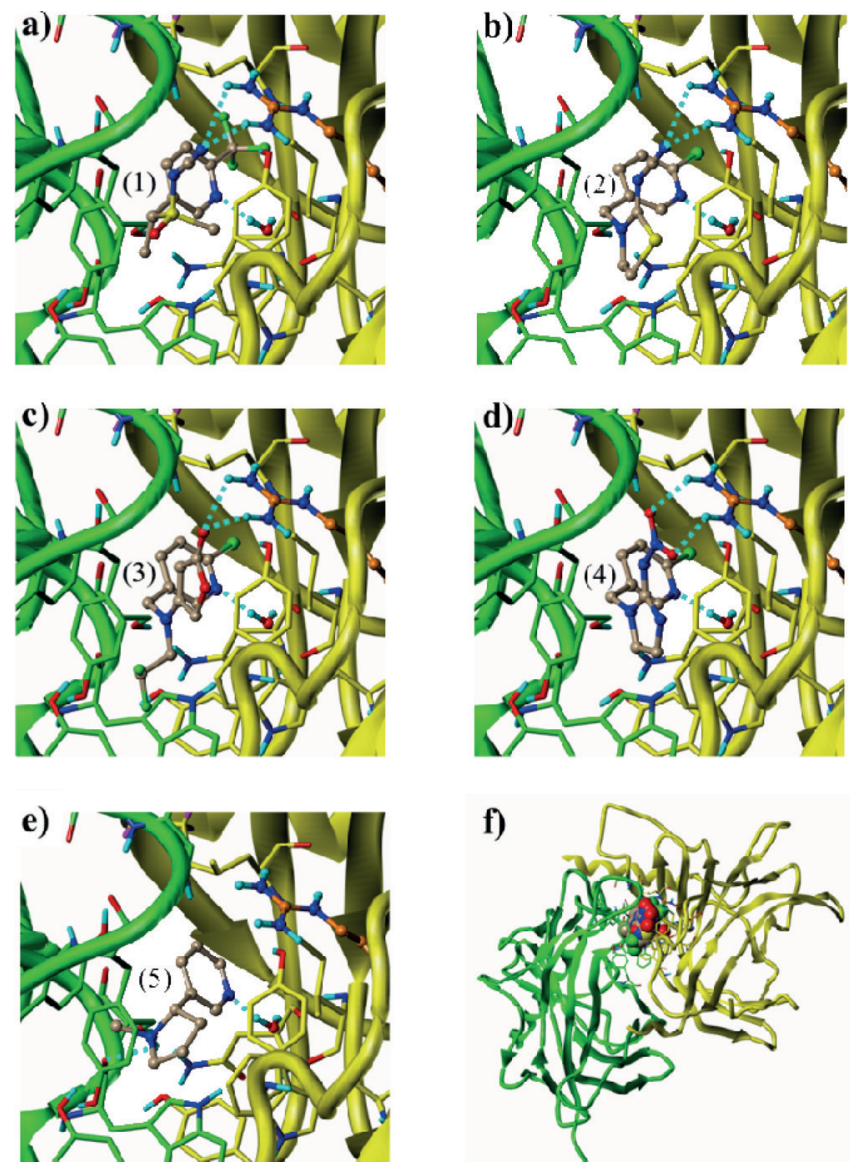

Figure 2. a-e) Binding mode hypothesis derived from docking of ligands 15 into a homology model of sensitive aphid nAChR. f) An overview of the model in the same orientation as in the other panels, with an overlay of all five agonists in space-fill representation. In all panels, yellow ribbons represent the $\beta_{1}$ subunit model, and the consensus model of the $\alpha_{1-5}$ subunit is shown in green. 
from loop $\mathrm{D}$ with the $\mathrm{N}$-cyanimino function of $\mathbf{1}$ and $\mathbf{2}$ with the 4-aminofuran-2(5H)-one group of 3 and with the $\mathrm{N}$-nitroimino group of 4, respectively. Trivially, this attractive, stabilizing interaction is not present in the case of nicotine (5). The distance between the protonated nitrogen atom in nicotine and the central carbon atom in the guanidinium head of arginine is in the order of $8 \AA$ (see the Supporting Information). As Coulomb-potential interactions scale with the inverse of the distance only and are, thus, far reaching, there will be a weak, yet noticeable repulsive force. Thus, the arginine residue from loop D strengthens the binding of the synthetic agonists, which are capable of forming $\mathrm{H}$-bridges, whereas in the case of nicotine, the presence of the same arginine residue can only negatively contribute to the binding strength.

Without going into further detail, it is notable that in resistance mutants of green peach aphid (Myzus persicae Sulzer) this charged amino acid is replaced by an uncharged threonine residue, just as in the respective vertebrate $n A C h R$ subunits (see the Supporting Information). In effect, this results in a loss of a decisive stabilizing interaction for $\mathbf{1}$ to $\mathbf{4}$, while at the same time removes a weak repulsive force in the case of nicotine. This is in line with the observed increased insecticidal efficacy as well as the beneficial mammalian toxicological profile of the synthetic agonists in contrast to that of nicotine. ${ }^{[11-13]}$ Further evidence derives from the observation that the biological profile of desnitro-imidacloprid, a derivative of imidacloprid missing the nitro group, resembles that of nicotine. ${ }^{[14]}$

From the docking poses, two further interactions can be speculated on that would be present in the binding modes of $\mathbf{3}$ and $\mathbf{5}$ only: a direct hydrogen bridge from the protonated nitrogen atom in nicotine (5) to the backbone of the cysteine loop on the $\alpha$ subunit and a hydrogen-bond-like interaction between the fluorine atom in the $\mathrm{N}$-(2,2-difluoroethyl) unit of flupyradifurone (3) as an acceptor and a hydroxy function of a tyrosine from the same loop in the $\alpha$ subunit as a donor. Depending on the chirality of the sulfur atom in sulfoxaflor (1), one may also wonder about the possibility of a hydrogen bridge towards the sulfoximine oxygen atom; however, the distance of this oxygen atom to any donor in the active site is too large $(>4 \AA)$. Table 2 provides an overview of the interactions in the model of sensitive wildtype insect $n A C h R$.

\subsection{Simplified Model Systems}

Comparing just the binding poses, or an alignment of 1-5 derived from their orientation in the active site, the five ligands

\begin{tabular}{|c|c|c|c|c|c|}
\hline & $\begin{array}{l}\beta \text { Suk } \\
\mathrm{H}-\text { bo } \\
\mathrm{H}_{2} \mathrm{O}\end{array}$ & $\begin{array}{l}\text { unit } \\
\text { id to: } \\
\text { Arg }\end{array}$ & $\begin{array}{l}\alpha \text { Subunit } \\
\text { "Aromatic } \\
\text { interaction" }\end{array}$ & $\begin{array}{l}\text { H-bond to: } \\
\text { backbone }\end{array}$ & Tyr \\
\hline sulfoxaflor (1) & yes & yes, M1 & yes, M1 & no & no \\
\hline thiacloprid (2) & yes & yes, M2 & yes, M2 & no & no \\
\hline imidacloprid (4) & yes & yes, M4 & yes, M4 & no & no \\
\hline flupyradifurone (3) & yes & yes, M3 & yes, M3 & no & yes, M7 \\
\hline nicotine (5) & yes & No & yes, M5 & yes, M6 & no \\
\hline
\end{tabular}

seem to behave in a strikingly similar fashion. To dig deeper into the nature of the binding modes, we went for a topological analysis at the level of electron density by QTAIM. To do so, drastic simplification towards model systems was necessary. The procedure on how to arrive at these model systems is outlined in Figure 3 by taking agonist 4 as an example. Only a min-

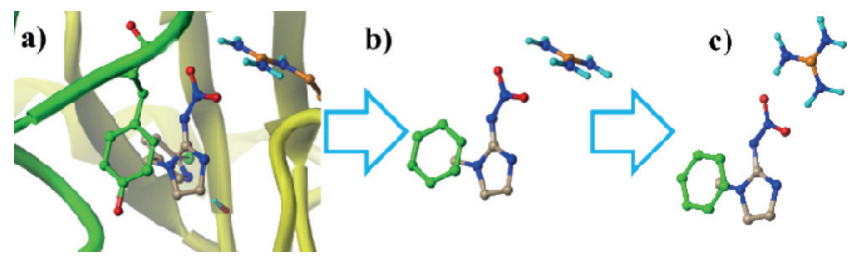

Figure 3. Outline of the procedure of how to arrive at the model systems, illustrated with imidacloprid (4) as an example. a) Binding mode in the $n A C h R$ model, along with two key residues of the binding pocket. b) Reduction to a head group model. c) Result of geometry optimization. See text for details.

imal set of residues is kept to represent the key anchoring interactions. These are: 1) one tyrosine, which happens to be the aromatic amino acid that is closest to the respective part of all five agonists, and 2) the arginine residue interacting with the head group (Figure $3 \mathrm{a}$ ). The pyridine systems of the agonists binding to loop $D$ through the conserved water molecule were then entirely removed. This was done as we did not expect any significant differences between the five agonists with respect to this rather ordinary hydrogen bond (a simple model
(6)<smiles>CS(C)(=O)=NC#N</smiles>

(8)<smiles>CN(C)C1=CC(=O)OC1</smiles>

(11)

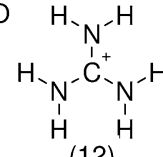
(12)<smiles>N#CN=C1SCCN1N</smiles>

(11)
(12)

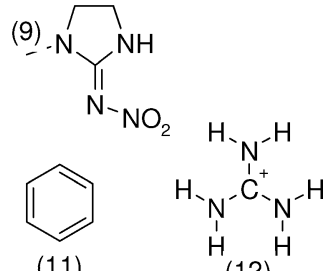

(11)
(12)<smiles>COc1ccccc1</smiles>

Figure 4. Model systems representing the core interactions of the head groups of agonists $\mathbf{1 - 5}$. 
system consisting of a pyridine-water complex is shown in the Supporting Information).

The tyrosine and arginine residues are represented by benzene (11) and the guanidinium cation (12), respectively (Figure $3 \mathrm{~b}$ and Figure 4). Starting from the coordinates of these atoms as observed in the binding mode model, the systems were saturated with hydrogen atoms and optimized by using density functional theory (Figure $3 \mathrm{c}$ ). During the optimization, the position of the guanidinium cation relative to the head group changes significantly; however, the relative orientation of the phenyl group stays more or less the same. The same procedure was used for synthetic agonists 1-3; in the case of nicotine (5), the final model system consists only of benzene (11) and protonated $\mathrm{N}$-methylpyrrolidine (10). Figure 4 provides an overview of the model systems, denoted models M1 to M5 in analogy to agonists $\mathbf{1}$ to $\mathbf{5}$.

To elucidate the interactions also specific to $\mathbf{3}$ and $\mathbf{5}$, two further model systems were created, M6 and M7 in Figure 5. Model M6 consists of protonated $N$-methylpyrrolidine (10), which forms a hydrogen bond towards the carbonyl function of formamide 13; M6 shall represent the hydrogen bridge from protonated nicotine (5) to the backbone discussed earlier in the context of docking poses.

The intention of model M7, finally, is to investigate potential interactions of the $\mathrm{N}$-(2,2-difluoroethyl) group of flupyradifurone (3) with neighboring tyrosine residues from the $\alpha$ subunit. M7 reduces this system to difluoromethane (14) and phenol (15). As already stated, the geometry optimizations of all model systems were performed by starting from the respective docking poses. Although changes in the relative orientation of the participating atoms and molecules in models M1-M6 were modest, small changes in the starting geometries resulted in significantly different geometries in M7. We selected only the resulting geometries that were relatively close to those observed in the parental docking pose; only one of these is discussed here. Further geometries and their QTAIM graphs can be found in the Supporting Information. We will only carefully discuss this system here to avoid over-interpretation in inferring from model M7 to the situation in the binding site.

\subsection{QTAIM Analysis of Model Systems}

The results obtained from Bader analysis are summarized in Fig- ures 6 and 7 and Table 3. The QTAIM graphs in these figures show nuclear critical points as larger spheres with colors encoding the respective element using the de facto standard color coding (C: gray, H: white, N: blue, O: red, S: yellow, halogens: green). Bond, ring, and cage critical points are shown as small spheres in green, red, and orange, respectively. Critical points associated to a "nonbonded" interaction, that is, an interaction that is not represented by any bond in the Lewis formulae in Figures 4 and 5, are highlighted by yellow circles. Bond critical paths are shown as gray tubes; broken tubes are used if the electron density at the respective bond critical point is below $0.025 \mathrm{au}$. The interaction of the head-group models with the guanidinium cation (12) in models M1-M4 are all characterized by two bond critical points and one ring critical point, which corresponds to two hydrogen bonds. In the

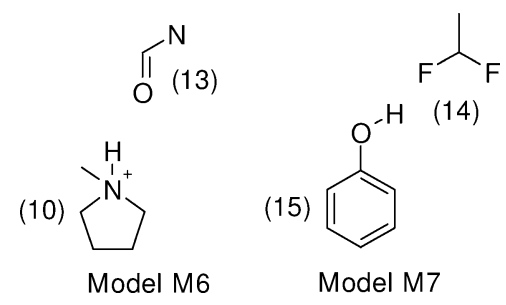

Figure 5. Two other model systems representing the interactions that are present only in the cases of agonists 3 and $\mathbf{5}$ : $\mathrm{H}$-bond of protonated $\mathbf{5}$ to the backbone (model M6) and $\mathrm{H}$-bond-like interaction of the $\mathrm{N}$-(2,2-difluoroethyl) group of 3 with tyrosine (model M7).
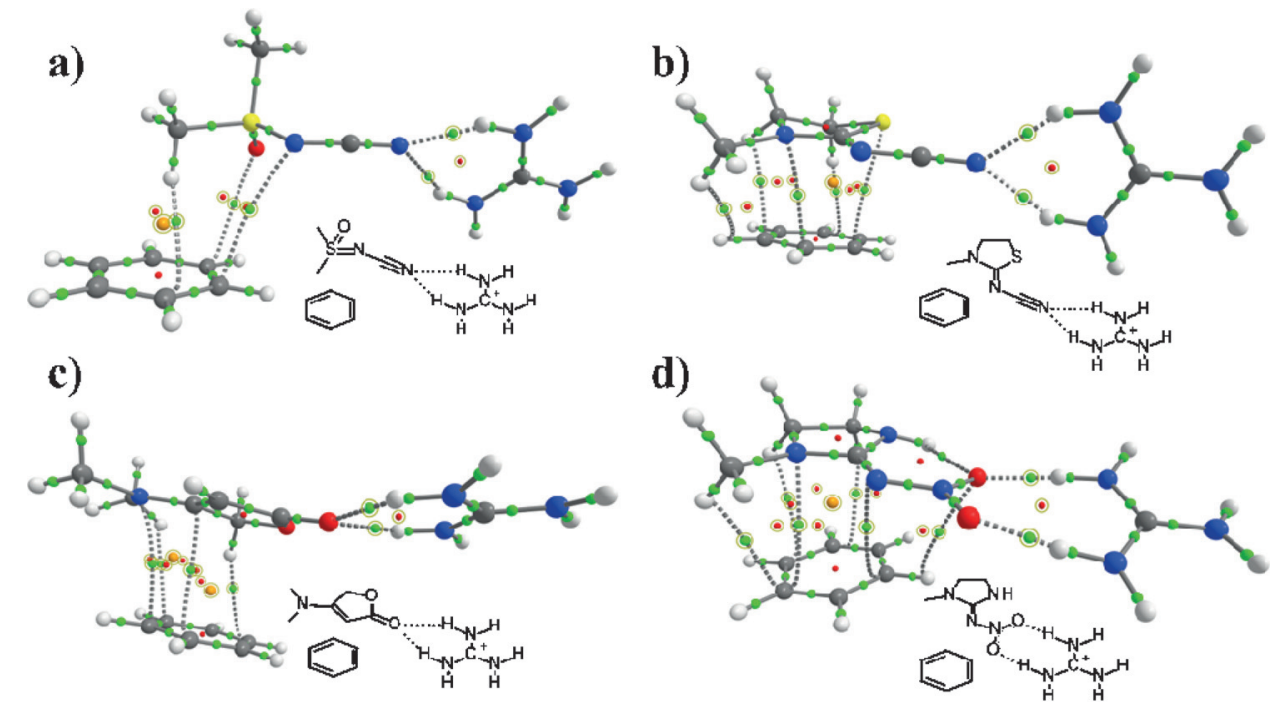

d)
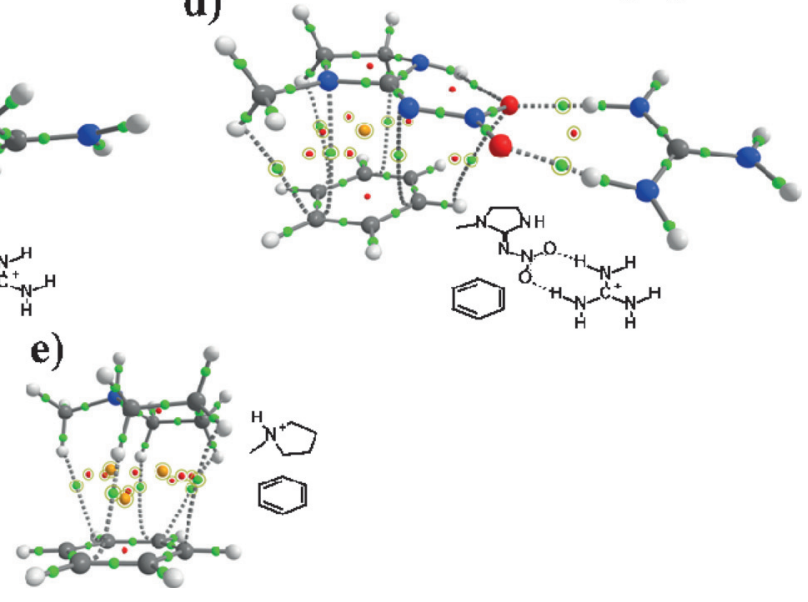

Figure 6. QTAIM graphs of the model systems from Figure 4. To facilitate reading, a schematic drawing of each system is provided next to the QTAIM graphs. Bold gray lines denote bond critical paths; those paths associated to BCPs with a charge density below 0.025 au are shown as dashed lines. BCPs are shown as green spheres, RCPs in red, CCPs in orange. CPs belonging to nonbonded interactions are highlighted by yellow circles. See Table 3 for charge densities of these CPs. 

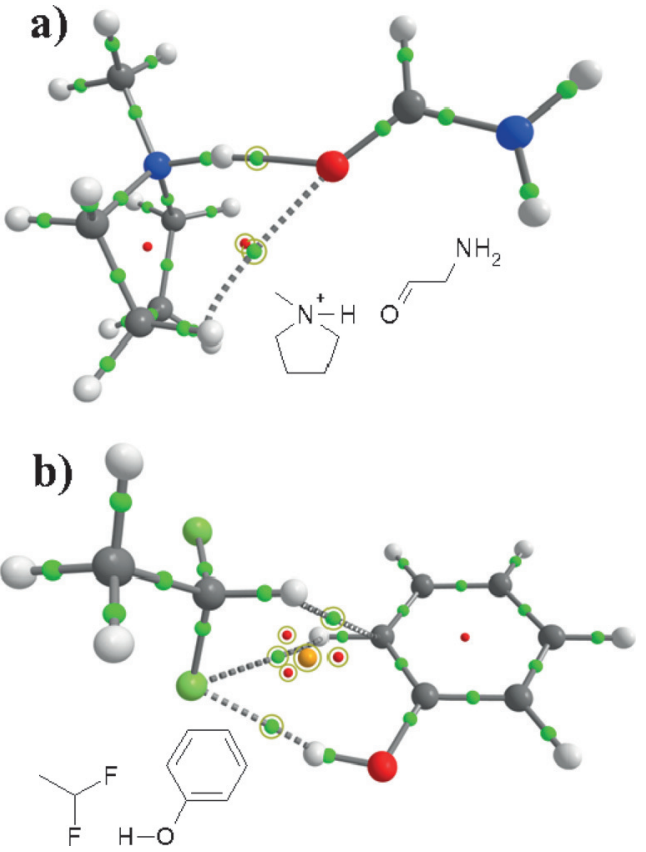

Figure 7. QTAIM graphs of model systems a) M6 and b) M7. See caption of Figure 6 for details.

model systems $M 1, M 2$, and $M 3$, three atoms are involved, two hydrogen atoms of the guanidinium cation (12) and just the "tip" of the $\mathrm{N}$-cyanimino functions $(\mathrm{NC}-\mathrm{N}=$ ) in models $\mathrm{M} 1$ and M2 or the 4-aminofuran-2(5H)-one (carbonyl oxygen atom) in model M3. The nitro group in model M4 also forms two hydrogen bonds, but these involve two pairs of atoms. In terms of electron density, the similarity of models $M 1$ and $M 2$, which both contain a $\mathrm{N}$-cyanimino moiety, is not surprising. However, that the 4-aminofuran-2(5H)-one in model M3 not only shares similar topology with models M1 and M2 but also similar electron densities of the critical points is perhaps more surprising.

Compared to the first three models, the interaction of the nitro group and the guanidinium cation (12) in model M4 accumulates somewhat more electron density in the bond critical points and somewhat less in the ring critical point. The sum over the electron density of all three critical points is clearly the largest for the nitro group, which reflects the expected high strength of this interaction. With regard to this interaction, sulfoxaflor (1) and thiacloprid (2) appear as a pair, and flupyradifurone (3) is the nearest neighbor and imidacloprid (4) is only a little further away.

Whereas the QTAIM analysis has more or less reproduced what an educated guess might have also predicted, the interactions of the model systems with the $\pi$ systems of the aromatic rings yield more discriminating features. Most strikingly in terms of topology, models M1, M2, and M4 exhibit just one cage critical point, whereas the 4-aminofuran-2(5H)-onephenyl interaction in model M3 bears two CCPs, and the $N$ methylpyrrolidinium-phenyl interaction in model M5 is characterized by three CCPs. As a result, models M3 and M5 collect more charge density in cage critical points than the other model systems. Model M5 is the only system involving interaction of the phenyl $\pi$ system with a formally charged partner, which is why the sum over the charge densities over all the CPs is largest for this model. Upon comparing the models in terms of the sums over the charge densities at the BCPs, RCPs, and CCPs, models M2 and M4, representing thiacloprid (2) and imidacloprid (4), form by far the closest pair (Eucledian distance of $0.005 \mathrm{au}$ in terms of the sums of charge densities in Table 3). The nearest neighbor with regard to the interaction to the phenyl group of model M5 (nicotine) is model M3 [flupyradifurone, (3)], with an Eucledian distance of 0.012 au.

In model system M6, there is a bond critical point connecting the $\mathrm{NH}$ group of the protonated $\mathrm{N}$-methylpyrrolidine system (10) to the carbonyl oxygen atom of formamide (13). The electron density associated to this BCP is the largest observed for any other BCP in this study $(0.057 \mathrm{au})$. This value is well above the threshold of 0.025 au used in the graphs in Figures 6 and 7 to denote "weak" bonds. It can be expected that the hydrogen bond connecting protonated nicotine (5) to the backbone of the cysteine loop of the $\alpha$ subunit will be a particularly strong one.

\begin{tabular}{|c|c|c|c|c|c|c|c|c|c|c|c|c|}
\hline \multicolumn{3}{|c|}{ Model system ${ }^{[a]}$} & \multicolumn{3}{|c|}{ Bond critical points } & \multicolumn{3}{|c|}{ Ring critical points } & \multicolumn{3}{|c|}{ Cage critical points } & \multirow{3}{*}{$\begin{array}{l}\text { All critical points } \\
\Sigma \rho_{\mathrm{i}} \\
0.045\end{array}$} \\
\hline & & & $\#$ & $\Sigma \rho_{\mathrm{i}}$ & $\rho_{1}$ range & $\#$ & $\Sigma \rho_{\mathrm{i}}$ & $\rho_{1}$ range & $\#$ & $\Sigma \rho_{\mathrm{i}}$ & $\rho_{1}$ range & \\
\hline M1 & (6) & (11) & 3 & 0.020 & $0.004-0.011$ & 3 & 0.017 & $0.004-0.009$ & 1 & 0.008 & & \\
\hline M2 & (7) & (11) & 5 & 0.039 & $0.006-0.011$ & 5 & 0.028 & $0.005-0.006$ & 1 & 0.005 & & 0.072 \\
\hline M3 & (8) & (11) & 4 & 0.032 & $0.007-0.011$ & 5 & 0.033 & $0.005-0.008$ & 2 & 0.012 & $0.005-0.008$ & 0.078 \\
\hline M4 & (9) & (11) & 6 & 0.042 & $0.004-0.010$ & 6 & 0.032 & $0.004-0.006$ & 1 & 0.003 & & 0.078 \\
\hline M5 & (10) & (11) & 5 & 0.041 & $0.006-0.011$ & 7 & 0.040 & $0.005-0.008$ & 3 & 0.016 & $0.004-0.008$ & 0.098 \\
\hline M1 & (6) & (12) & 2 & 0.062 & $0.030-0.031$ & 1 & 0.012 & & & & & 0.074 \\
\hline M2 & (7) & (12) & 2 & 0.065 & $0.032-0.033$ & 1 & 0.012 & & & & & 0.077 \\
\hline M3 & (8) & (12) & 2 & 0.074 & $0.031-0.043$ & 1 & 0.012 & & & & & 0.086 \\
\hline M4 & (9) & (12) & 2 & 0.084 & $0.04-0.044$ & 1 & 0.007 & & & & & 0.091 \\
\hline M6 & (10) & (13) & 2 & 0.062 & $0.005-0.057$ & 1 & 0.005 & & & & & 0.067 \\
\hline M7 & (14) & (15) & 3 & 0.030 & $0.006-0.018$ & 3 & 0.016 & $0.005-0.006$ & 1 & 0.005 & & 0.051 \\
\hline
\end{tabular}

[a] Labeling refers to Figure 4 in the main text and Scheme S1; for example, in the first row, M1 (6) (11) shows properties of critical points belonging to the nonbonded interaction of the sulfoxaflor head group (6) with a phenyl moiety (11) of model M1. 
Just from the distances in the docking pose of flupyradifurone (3) in the active site, it seems possible that the $\mathrm{N}$-(2,2-difluoroethyl) moiety of $\mathbf{3}$ forms hydrogen-bridge-like interactions to one or even two of the adjacent tyrosine residues from the $\alpha$ subunit. Such an interaction is not possible for the other four agonists investigated herein. The nature of hydrogen bonds involving fluorine is delicate and has been debated for decades. ${ }^{[55-57]}$ Meanwhile, there is sufficient evidence for a range of nonbonded interactions mediated by fluorine. ${ }^{[58-60]}$

As already stated, optimization of model system M7 is very sensitive to small changes in the starting geometry, which leads to different relative orientations of the interacting partners. Other model systems related to M7 and their QTAIM graphs are provided in the Supporting Information.

However, there are some trends common to all individual geometries from calculations of model system M7. There are always three bond critical points connecting the difluoroethyl moiety to phenol. The BCP with the highest charge density is found between one fluorine atom and the hydroxy hydrogen atom, with charge densities ranging from 0.014 to 0.018 (see the Supporting Information). Interestingly, the maximum of these values is found just in the geometry shown in Figure 7, which is the geometry that comes closest to the respective docking pose of the full system. The two remaining BCPs carry far less electron density (in the order of 0.005-0.006 au). One of these two always connects the same fluorine atom, which already interacts with the hydroxy hydrogen atom, to a second hydrogen atom of phenol in the meta position. Finally, the third BCP connects the terminal hydrogen atom of the $\mathrm{N}-(2,2-$ difluoromethyl) group to either the respective carbon atom in the meta position, as is the case also in Figure 7, or the phenol oxygen atom. It has been frequently observed that fluorine plays a special role in structure-activity relationships. ${ }^{[61]}$ Also in the case of flupyradifurone, the $\mathrm{N}$-difluoroethyl group can clearly contribute significantly to its binding.

\section{Conclusions}

Starting from a homology model of the active site of the nicotinic acetylcholine receptor (nAChR) of sensitive pest insects, such as aphids (here, Myzus persicae), a hypothesis for the mode of binding was created for five small-molecule agonists of the $n A C h R$, representing all four chemical subclasses of group 4 in the Insecticide Resistance Action Committee (IRAC) mode of action (MoA) classification scheme. Docking poses of these agonists in the active site of the homology model revealed two major interactions that are common across all investigated compounds: 1) a water-mediated hydrogen bridge towards the backbone of the $\beta$ subunit of the receptor. 2 ) an interaction with a conserved cluster of aromatic residues from the $\alpha$ subunit. Interactions 1 and 2 result in nice spatial alignment of all five agonists within the active site. 3) The four synthetic agonists carry a head group that forms a hydrogen bridge towards a charged arginine residue from the $\beta$ subunit, which is known to be crucial for high-affinity binding and pest sensitivity. Interaction 3 is not only absent for the vertebrate toxic plant derived alkaloid nicotine, but is replaced by a weak electrostatic repulsion. On the other hand, if protonated (see 5), nicotine is the only compound capable of also forming a strong hydrogen bond to the backbone of the $\alpha$ subunit (interaction 4). Among the synthetic agonists, flupyradifurone (3) can interact with tyrosine residues from the $\alpha$ subunit not only through its head group (referred to as interaction 2 above), but also in a hydrogen-bridge-like fashion to the hydroxy functions of these amino acids through its $\mathrm{N}$-(2,2-difluoroethyl) function (interaction 5). The anticipated joint effect of these interactions is in line with the observed differences of nicotine, on the one hand, and synthetic agonists 1-4, on the other hand, with respect to their insecticidal efficacy as well as vertebrate safety and toxicity.

Small model systems were created to further investigate the just summarized interactions. At the level of calculated electron densities and their Laplacians, all interactions derived from the docking poses are reflected in the electron density. Moreover, the analysis revealed differences in the topology of binding between nicotine, sulfoxaflor (1), flupyradifurone (3), and the two neonicotinoids (i.e., 2 and 4 ) even for the interactions present in all binding motives, which thus principally supports the classification of these group 4 compounds into four distinct chemical subgroups within the IRAC MoA classification scheme.

The combined use of docking poses and analysis by quantum theory of atoms in molecules of small-model systems can thus lead to greater insight into binding mode hypotheses. The limited effort required to refine and use these methods makes them suitable tools not only for an a posteriori analysis, but also for applications in the design of new active ingredients, not restricted to agonists of the insect nAChR. In the future, the method could also be used to better understand sensitivity and selectivity aspects across different insect species as well as in comparison to vertebrates.

\section{Computational Details, Methods, and Programs}

Homology models of nAChR subunits were created by using the Orchestrar suite of programs as implemented in SYBYL- ${ }^{[38]}$ which essentially is an implementation of Blundell's structure assisted sequencing approach, also known as threading. ${ }^{[39,40,41]}$ Clash regions in resulting dimers were refined with the molecular dynamics tools of SYBYL-X by using the Amber7 FF02 force field ${ }^{[43]}$.

All quantum chemical calculations were performed by using Turbomole $6.5 \cdot{ }^{[44,45]}$ The level of theory was density functional theory, ${ }^{[29]}$ making use of multipole acceleration and the resolution of identity approximation. ${ }^{[46,47]}$

The Becke-Perdew (B-P86) functional was used, that is, a combination of Becke (B88) exchange ${ }^{[48]}$ and Perdew (P86) correlation functionals. ${ }^{[49]}$ Ahlrich's def2-TZVPP basis sets ${ }^{[50]}$ were employed at all atoms. Basis sets with fewer polarization functions, for example, def2-SVP, did not perform well in the optimizations, as they had a tendency to ignore the weak interactions. B-P86//def2-TZVPP level of theory provides a good balance of accuracy as well as practicability in terms of computing time and resources. ${ }^{[31]}$ Grimme's D3 dispersion correction ${ }^{[51]}$ was used to overcome the notorious weakness of DFT in describing weakly bonded systems. 
In our hands, this method has proven to be robust and reliable and delivers results that in many cases can compete with perturbation theory MP2.

QTAIM analysis of the resulting wavefunctions and electron densities was performed in AIMStudio 14.06.21. ${ }^{[52]}$

All graphics were created either with SYBYL-X ${ }^{[38]}$ or AIMAll 14.06.21.

\section{Acknowledgements}

The authors would like to thank Ralf Nauen, Peter Jeschke, Robert Velten, Torsten Bürger, and Michael Schindler for valuable discussions. We are grateful to Mark James Ford for carefully proofreading the manuscript.

Keywords: density functional calculations - ion channels molecular modeling $\cdot$ protein models $\cdot$ quantum chemistry

[1] A. Karlin, Nat. Rev. Neurosci. 2002, 3, 102-114.

[2] N. S. Millar, C. Gotti, Neuropharmacology 2009, 56, 237-246.

[3] D. B. Sattelle, A. K. Jones, B. M. Sattelle, K. Matsuda, R. Reenan, P. C. Biggin, BioEssays 2005, 27, 366-367.

[4] S. M. Sine, A. G. Engel, Nature 2006, 440, 448-455.

[5] S. H. Thany, G. Lenears, V. Raymond-Delpech, D. B. Sattelle, B. Lapid, Trends Pharmacol. Sci. 2007, 28, 14-22; N. S. Millar, I. Denholm, Invertebr. Neurosci. 2007, 7, 53-66.

[6] H. Breer, D. B. Sattelle, J. Insect Physiol. 1987, 33, 771-790.

[7] A. K. Jones, V. Raymond-Delpech, S. H. Thany, M. Gauthier, D. B. Sattelle, Genome Res. 2006, 16, 1422-1430.

[8] E. D. Gundelfinger, R. Schulze in Handbook of Experimental Pharmacology: Neuronal Nicotinic Receptors (Eds.: F. Clementi, D. Fornasari, C. Gotti), Springer, Berlin, 2000, Vol. 44, pp. 497-521.

[9] AG Chem. New Compound Rev Vol. 30, Agranowa, Orpington, 2012.

[10] Overview Report: Neonicotinoids and the Health of Honey Bees in Australia, Australian Pesticides and Veterinary Medicines Authority (APVMA), Kingston, February, 2014.

[11] P. Jeschke, R. Nauen, M. E. Beck, Angew. Chem. Int. Ed. 2013, 52, $9464-$ 9485; Angew. Chem. 2013, 125, 9640-9662.

[12] A. Elbert, R. Nauen in Insect Pest Management, Field and Protection Crops (Eds.: A. R. Horowitz, I. Ishaaya), Springer, Berlin, 2004, pp. 29-57.

[13] A. Cantoni, H. W. Schmidt, J. Gilli, Pflanzenschutz-Nachr. Bayer 2001, 54, $353-368$.

[14] M. Tomizawa, J. E. Casida, Annu. Rev. Entomol. 2003, 48, 339-364.

[15] Th. C. Sparks, R. Nauen, Pestic. Biochem. Physiol. 2015, 121, 122-128.

[16] Th. C. Sparks, Pestic. Biochem. Physiol. 2013, 107, 1-7.

[17] P. Jeschke, R. Nauen, O. Gutbrod, M. E. Beck, S. Matthiesen, M. Haas, R. Velten, Pestic. Biochem. Physiol. 2015, 121, $31-38$.

[18] R. Nauen, P. Jeschke, R. Velten, M. E. Beck, U. Ebbinghaus-Kintscher, W. Thielert, K. Wölfel, M. Haas, K. Kunz, G. Raupach, Pest Manag. Sci. 2015, $71,850-862$.

[19] N. Unwin, A. Miyazawa, J. Li, Y. Fujiyoshi, J. Mol. Biol. 2002, 319, $1165-$ 1176.

[20] K. Brejc, W. J. van Dijk, R. V. Klaasen, M. Schurmans, J. van der Oost, A. B. Smit, T. K. Sixma, Nature 2001, 411, 269-276.

[21] M. Tomizawa, D. Maltby, T. T. Talley, K. A. Durkin, K. F. Medzihradszky, A. L. Burlingame, P. Taylor, J. E. Casida, Proc. Natl. Acad. Sci. USA 2008, $105,1728-1732$.

[22] M. Tomizawa, J. E. Casida, Acc. Chem. Res. 2009, 42, 260-269.

[23] P. H. Celie, R. V. Klaasen, S. E. van Rossum-Fikkert, R. van Elk, P. van Nierop, A. B. Smit, T. K. Sixma, J. Biol. Chem. 2005, 280, 26457- 26466.

[24] Ch. Ulens, A. Akdemir, A. Jongejan, R. van Elk, S. Bertrand, A. Perrakis, R. Leurs, A. B. Smit, T. K. Sixma, D. Bertrand, I. J. P. de Esch, J. Med. Chem. 2009, 52, 2372-2383.
[25] G. Klebe, Drug Design: Methodology, Concepts, and Mode-of-Action, Springer, Berlin, 2013.

[26] J. B. Cross, D. C. Thompson, B. K. Rai, J. Ch. Baber, K. Y. Fan, Y. Hu, Ch. Humblet, J. Chem. Inf. Model. 2009, 49, 1455-1474.

[27] M. E. Beck, unpublished results.

[28] G. N. Lewis, J. Am. Chem. Soc. 1916, 38, 762-785

[29] R. G. Parr, W. Yang, Density Functional Theory of Atoms and Molecules, Clarendon Press, New York, 1989.

[30] K. Fukui, Science 1982, 218, 747-754.

[31] M. E. Beck, J. Chem. Inf. Model. 2005, 45, 273-282.

[32] R. F. W. Bader, Atoms in Molecules: A Quantum Theory, Oxford University Press, Oxford, 1990

[33] R. F. W. Bader, Acc. Chem. Res. 1985, 18, 9-15.

[34] The Quantum Theory of Atoms in Molecules: From Solid State to DNA and Drug Design (Eds.: Ch. F. Matta, R. J. Boyd), Wiley-VCH, Weinheim, 2007.

[35] E. Espinosa, E. Molins, C. Lecomte, Chem. Phys. Lett. 1998, 285, $170-$ 173.

[36] E. Espinosa, E. Molins, J. Chem. Phys. 2000, 113, 5686-5694.

[37] M. P. Waller, A. Robertazzi, J. A. Platts, D. E. Hibbs, P. A. Williams, J. Comput. Chem. 2006, 27, 491-504.

[38] SybyIX2.1.1, L.P. Certara, St. Louis, MO, 2014.

[39] J. Shi, T. L. Blundell, K. Mizuguchi, J. Mol. Biol. 2001, 310, 243- 257.

[40] Orchestrar in SybyIX2.1.1, L.P. Certara, St. Louis, MO, 2014.

[41] P. De Bakker, A. Bateman, D. F. Burke, R. N. Miguel, K. Mizuguchi, J. Shi, H. Shirai, T. L. Blundell, Bioinformatics 2001, 17, 748-749.

[42] T. Bürger, MOCCA: Conformational Analysis Combining Monte Carlo and Simulated Annealing Techniques, unpublished results.

[43] D. A. Case, D. A. Pearlman, J. W. Caldwell, T. E. Cheatham III, J. Wang, W. S. Ross, C. L. Simmerling, T. A. Darden, K. M. Merz, R. V. Stanton, A. L. Cheng, J. J. Vincent, M. Crowley, V. Tsui, H. Gohlke, R. J. Radmer, Y. Duan, J. Pitera, I. Massova, G. L. Seibel, U. C. Singh, P. K. Weiner, P. A. Kollman, AMBER 7, University of California, San Francisco, 2002.

[44] R. Ahlrichs, M. Baer, M. Haeser, H. Horn, C. Koelmel, Chem. Phys. Lett. 1989, 162, 165- 169.

[45] O. Treutler, R. Ahlrichs, J. Chem. Phys. 1995, 102, 346-354.

[46] K. Eichkorn, O. Treutler, H. Oehm, M. Häser, R. Ahlrichs, Chem. Phys. Lett. 1995, 240, 283-290.

[47] K. Eichkorn, O. Treutler, H. Oehm, M. Häser, R. Ahlrichs, Chem. Phys. Lett. $1995,242,652-660$.

[48] A. D. Becke, Phys. Rev. A 1988, 38, 3098-3100.

[49] J. Perdew, Phys. Rev. B 1986, 33, $8822-8824$.

[50] K. Jankowski, R. Becherer, P. Scharf, R. Ahlrichs, J. Chem. Phys. 1985, 82, $1413-1419$.

[51] S. Grimme, J. Anthony, S. Ehrlich, H. Krieg, J. Chem. Phys. 2010, 132, $154104-154119$.

[52] T. A. Keith, AIMAll (Version 14. 06. 21), TK Gristmill Software, Overland Park, KS, 2014.

[53] M. Ihara, T. Okajima, A. Yamashita, T. Oda, K. Hirata, H. Nishiwaki, T. Morimoto, M. Akamatsu, Y. Ashikawa, S. Kuroda, R. Mega, S. Kuramitsu, D. B. Sattelle, K. Matsuda, Invertebr. Neurosci. 2008, 8, 71-81.

[54] T. T. Talley, M. Harel, R. E. Hibbs, Z. Radic, M. Tomizawa, J. E. Casida, P. Taylor, Proc. Natl. Acad. Sci. USA 2008, 105, 7606-7611.

[55] C. Bissantz, B. Kuhn, M. Stahl, J. Med. Chem. 2010, 53, $5061-5084$.

[56] J. D. Dunitz, R. Taylor, Chem. Eur. J. 1997, 3, 89-98.

[57] S. Purser, P. R. Moore, S. Swallow, V. Gouverneur, Chem. Soc. Rev. 2008, $37,320-330$.

[58] H. Plenio, ChemBioChem 2004, 5, 650-655.

[59] H. Adams, S. L. Cockroft, C. Guardigli, Ch. A. Hunter, K. R. Lawson, J. Perkins, ChemBioChem 2004, 5, 657-665.

[60] J. A. Olsen, D. W. Banner, P. Seiler, B. Wagner, Th. Tschopp, U. ObstSander, M. Kansy, K. Müller, F. Diederich, ChemBioChem 2004, 5, 666675.

[61] P. Jeschke, ChemBioChem 2004, 5, 570-589.

Manuscript received: April 28, 2015

Final Article published: July 14, 2015 